\title{
THE USE OF PHYLOGENY IN BEHAVIOR AND ECOLOGY
}

\author{
F. STEPHEN Dobson \\ Museum of Zoology and Division of Biological Sciences \\ University of Michigan, Ann Arbor, MI 48109
}

Received July 13, 1984. Accepted July 12, 1985

The comparative analysis of characteristics of different species is extensively used in evaluating possible selective contexts for behavioral and ecological differences among species (e.g., Alexander et al., 1979; Blumer, 1979; Bradbury, 1981; Dobson, 1982; Greenwood, 1980; Ralls, 1976; Stearns, 1977; Wittenberger, 1976; Wittenberger and Tilson, 1980). Such analyses often explicitly or implicitly assume that when species resemble each other in some behavioral or morphological characteristic, the resemblance is due to independent appearance of the characteristic under study in each species in the analysis. This assumption can be (but rarely is) tested by examining evidence for independent evolution of characteristics, deduced from a well supported hypothesis of phylogeny. In this essay, I will explain how non-systematists in general, and behaviorists and ecologists in particular, can benefit from considering historical influences on the evolution of characteristics. I will also indicate problems that may be encountered in extracting a well supported phylogeny from the systematic literature.

\section{Historical Analysis}

The characteristics that contemporary species exhibit may have evolved in their recent environment, or they may be of relatively older evolutionary origin. That is, a group of related species may have derived characteristics independently or from a common ancestor. If a characteristic is obtained through descent from an ancestor, then in comparison with the ancestor the characteristic may be of greater, equal, or less utility to individuals in the descendent species in coping with their respective contemporary environments. Genes for the characteristic should remain in the genomes until a more beneficial alternative characteristic arises. In a group of contemporary species that share a particular characteristic, therefore, it might be difficult to ascertain just how beneficial the characteristic is in the respective environments of the species. It might be even more difficult (or impossible) to judge whether alternative characteristics that have never evolved in the group of species might be more beneficial than an existing characteristic. Thus, it might be misleading to assume that a characteristic is an adaptation to contemporary environments, and a characteristic that descends from a common ancestor to contemporary species cannot be assumed to be of "optimal" benefit to individuals.

In interspecific comparisons, sets of alternative characteristics are examined for correlations among themselves, or for correlations among characteristics and environments. If a group that shares a characteristic is also a monophyletic group, then there is an alternative to explanations of natural selection in recent (modern) environments: the characteristic might have evolved in a past environment, and it is retained because superior alternatives did not arise in the taxa. In an interspecific comparison in which monophyletic and character trait groups cooccur, the a priori historical origin hypothesis is an equally valid hypothesis compared to any explanation of superior function (i.e., superior adaptiveness) in recent environments.

Shared morphological characteristics in a monophyletic group are commonly thought to be influenced strongly by a common ancestry. For example, numerous characteristics of reproductive morphology of marsupial mammals are shared widely within the clade (the monophyletic group), and they are probably descended from a single ancestor and conserved over evolutionary time (Lillegraven, $1974,1975)$. Whether marsupial reproductive morphology is strongly constrained against change (Lillegraven, 1974, 1975) or highly adaptive in certain environments (Low, 1978), or both, it would be unsatisfactory to explain the distribution of reproductive characteristics between marsupial and placental mammals solely as the result of natural selection operating in recent environments. The historical origin hypothesis is corroborated by phylogenetic analysis in this case, and it should be considered a valid alternative to adaptive explanations.

The hypothesis of shared similarity due to shared history can also be applied to behavioral studies. For example, Greenwood (1980) found that characteristics of juvenile dispersal, with several important exceptions, differed between birds and mammals. In about $90 \%$ of bird species, juvenile males and females disperse equally, or juvenile females exhibit somewhat greater dispersal than juvenile males. In about $70 \%$ of mammal species, males are the predominant dispersing sex among juveniles. The hypothesis that the characteristics of these taxa might be shared among species because they share a common historical origin should be considered along with the adaptive hypotheses discussed by Greenwood (1980).

The historical origin hypothesis might also prove illuminating in population ecology. One of the most intensively studied questions in population ecology 
is why many species of microtine rodents exhibit "cyclic" or erratic outbreaks in population size every three to four years. Krebs and Myers (1974) argued that the widespread occurrence of fluctuating $\mathrm{mi}-$ crotine populations makes it likely that a single causal mechanism is responsible. Perhaps a propensity for cyclic fluctuations due to characteristics of individual microtine rodents (in particular environments) has been inherited by many microtine species from their common ancestor. Ecologists have recently emphasized causal mechanisms that are "intrinsic" to fluctuating microtine rodents, and they have assumed that the characteristics of individuals that result in changes in population size are adaptive in contemporary environments (e.g., Krebs, 1978; Lidicker, 1978). An influence of historical origin (an intrinsic factor) has not previously been suggested.

I will not present examples of the relevance of the historical origin hypothesis to the study of functional morphology, life history, or coevolution. Lauder $(1981,1982)$ presented methods for testing the hypothesis of historical influence on morphological form and argued that functional morphology should be viewed as an historical discipline. Stearns $(1983,1984)$ has indicated a strong influence of phylogeny on the evolution of life histories (but see Vitt and Seigel, 1985), and the influence of historical origin was applied to the evolution of ecological associations by Mitter and Brooks (1983) and Brooks (1985). These studies indicate a growing interest in the value of considering the influence of history in interspecific studies. Tests of historical influences need to be extended to studies of the evolution of behaviors and to additional subfields of ecology. Such tests can be used as an important first step in the design of research, in which groups of species that are most likely to exhibit adaptations to recent environments are chosen for testing evolutionary hypotheses.

The historical origin hypothesis can be tested by comparing the distribution of behavioral or ecological characteristics among species to a well supported phylogeny for the species. If the distribution of characteristics among species is inconsistent with the phylogeny, then several independent evolutionary events are required to explain the distribution of characteristics. Under these conditions, it is unlikely that the distribution of characteristics has been strongly influenced by historical origin.

\section{Four Methods}

I used this method in a recent examination of the occurrence of territoriality by adult males during the mating season in the sciurid rodents of North America (Dobson, 1984). I compared the occurrence of territoriality to the best supported phylogeny of sciurid species, and found that in the ground squirrels (genera Cynomys and Spermophilus), male territoriality probably arose independently more than once. Thus, I could reject the historical origin hypothesis as a major determinant of the occurrence of male territoriality and proceed to test hypotheses that invoke adaptation to recent environments.

A second method can be used to insure that patterns of characteristics among taxa are likely to be due primarily to historical origin. Alexander et al. (1979) examined the degree of polygyny and degree of sexual dimorphism in three distantly related taxa (pinnipeds, primates, and ungulates). Each taxon contained a wide range of variation in the characteristics of polygyny and sexual dimorphism, and the range of variation likely evolved independently at least three times. While a consideration of historical influences was not explicitly mentioned by Alexander et al. (1979), a primary influence of historical origin on the pattern of characteristics that they examined is unlikely.

Ridley (1983) suggested a third method for identifying independent evolution that assigns characteristics to ancestors by successive "outgroup" comparisons. Such inference of behavioral or ecological characteristics of ancestors, however, is not required for testing hypotheses of historical origin. As the previous two methods indicate, all that is required is evidence of the minimum number of independent evolutionary events (i.e., assignment of a hypothetical ancestral condition that minimizes potential character evolution). For example, Ridley (1983) states that his method cannot be used in cases like the study by Alexander et al. (1979), despite considerable evidence of independent evolution.

A fourth method that corrects comparative studies for phylogeny was developed by Felsenstein (1985). This statistical methodology has considerable barriers to practical application (discussed by Felsenstein), but it illustrates the difficulties involved in trying to separate environmental and historical influences on the characteristics of organisms. Until methods for partitioning these influences are available, methods that indicate when the influence of history is minimized or maximized will likely be most helpful to behaviorists, ecologists, and other functional biologists.

\section{Example}

The cladogram (a hypothetical phylogeny that shows speciation events, but not the time between speciation events) that behaviorists and ecologists use to test the common historical origin hypothesis will, in most cases, come from the literature. Unfortunately, the systematic classification of a group of animals cannot always be used to produce a cladogram. For example, prairie dogs, genus Cynomys, are thought to be a sister group to the ground squirrel subgenus Spermophilus (Black, 1963; Bryant, 1945; Hight et al., 1974; Nadler et al., 1971). The group formed by prairie dogs and this subgenus (a monophyletic group, which by definition includes all related species) is more distantly related to the other ground squirrel subgenera (see Fig. 1c and Table 1A). The genus Spermophilus, therefore, is paraphyletic (i.e., not all the related species are included in the genus). 
a.

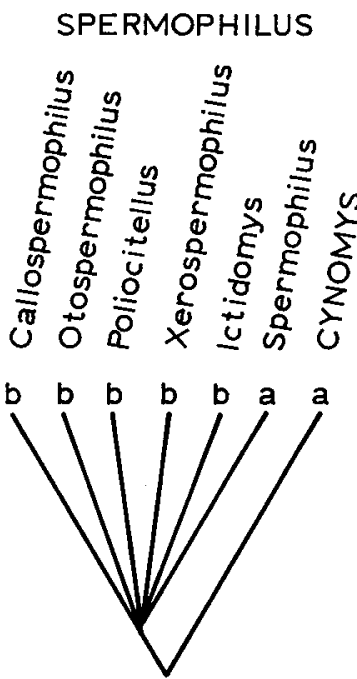

b.

c.

FIG. 1. a) A hypothetical phylogeny of ground squirrels, derived from the current classification system (see Table 1A). b) The hypothetical phylogeny derived from the monophyletic classification system in Table 1B. c) The phylogenetic relationships of the ground squirrels, modified from Bryant (1945). The genus Cynomys and the subgenera of the genus Spermophilus are shown. The placement of Cynomys is consistent with several systematic studies (Bryant, 1945; Black, 1963; Nadler et al., 1971; Hight et al., 1974). In a), b), and c), homologous behavioral characteristics $a$ (the run-jump) and $b$ (absence of the run-jump) are assigned to taxa (see text). Genera are completely capitalized and subgenera include lower case lettering.

Taxonomies that contain paraphyletic groups cannot be used to test hypotheses that invoke historical origin of behavioral or ecological characteristics. For example, I have observed a peculiarity in the running gait of black-tailed prairie dogs $(C$. ludovicianus) and Richardson's ground squirrels $(S$. richardsonii, subgenus Spermophilus). When fleeing from an observer (and, presumably, from terrestrial predators) individuals of these species often push the anterior part of the body off the ground with the forelegs, while continuing to run with the hind legs (in the prairie dog this behavior is associated with vocalization; King, 1955). I have not observed this behavior (the "run-jump") in observations of other ground squirrels, nor have I found mention of it in the ethological literature. Prairie dogs may be most closely related to Richardson's ground squirrels (Nadler et al., 1971), and they might share the run-jump behavior because it was present in a common ancestor.

The presence of the run-jump in the genera and subgenera of prairie dogs and ground squirrels follows the pattern of character state $a$ in Figure 1, and the absence of the run-jump (according to the current literature) follows the distribution of character state $b$. If the current systematic classification of ground squirrels and prairie dogs is used as a basis for comparative study of the run-jump behavior (Table 1A), the results would be inconsistent with respect to the best supported phylogeny (Fig. 1c). Using the phylogeny constructed from the current system of classification (Fig. 1a), more than one independent evolutionary origin of the runjump or its homolog (the absence of the run-jump) is required. A monophyletic system of classification (Table 1B) can be used to test the common historical origin hypothesis, and in this case only one independent evolutionary event is required (Fig. 1b). Note that some classifications do not allow testing of the common historical origin hypothesis (e.g., Table $1 \mathrm{C}$ is monophyletic, but it cannot be used to provide a relevant test in my example). In such cases, a more complete hypothetical phylogeny must be examined (e.g., Fig. 1c).

An adaptive scenario to explain the evolutionary origin of the run-jump behavior might be that it improves visual detection of terrestrial predators in prairie habitats, perhaps due to visual obstruction of tall grass. Because the historical origin hypothesis is supported, however, an investigation of the habitat of the ancestor would be required to test the scenario. If it were observed that the behavior occurs irrespective of the visual obstruction of the habitat, the scenario might be falsely rejected be- 
TABLE 1. Alternate systems of classification of the ground squirrels. The order of listed genera and subgenera has no special meaning. The current system (A) is paraphyletic, and it cannot be used to reconstruct a phylogeny that is consistent with Figure 1c. The monophyletic systems (B and C) can be used to reconstruct phylogenies that are consistent with Figure 1c. Monophyletic system II (C) is most consistent with both the phylogeny and the current generic and subgeneric designations.
A.
Current system
Genus Cynomys
Genus Spermophilus
Subgenus Spermophilus
Subgenus Ictidomys
Subgenus Poliocitellus
Subgenus Xerospermophilus
Subgenus Otospermophilus
Subgenus Callospermophilus
B. Monophyletic System I
Genus Cynomys
Subgenus Cynomys
Subgenus Spermophilus
Genus Ictidomys
Genus Poliocitellus
Genus Xerospermophilus
Genus Otospermophilus
Genus Callospermophilus
C. Monophyletic System II
Genus Cynomys
Subgenus Cynomys
Subgenus Spermophilus
Subgenus Ictidomys
Subgenus Poliocitellus
Subgenus Xerospermophilus
Subgenus Otospermophilus
Subgenus Callospermophilus

cause the behavior is not evolutionarily maintained as the scenario predicts. In this case, the possible historical inheritance of the characteristic renders the evolutionary scenario of origination untestable in recent environments.

\section{Conclusion}

Because some systematists produce taxonomies that do not reflect phylogeny (i.e., paraphyletic taxonomies), the behaviorist or ecologist cannot simply refer to a taxonomy to test historical origin hypotheses, without study of the method that produced the taxonomy. The safest procedure is to extract the best supported phylogeny from the systematic literature. Taxonomies designed by Hennigian cladists, however, should be very useful to behaviorists and ecologists. Cladists are primarily concerned with accurate inference of patterns of shared history, and their taxonomies can be expected to reflect the most accurate estimates of phy- logeny (see Eldredge and Cracraft, 1980; Wiley, 1981). Naturally, the strongest tests of historical origin hypotheses will be in groups in which systematists have little disagreement concerning hypothetical phylogeny.

\section{ACKNOWLEDGMENTS}

I owe A. G. Kluge and P. Myers special thanks for patient discussions of the ideas presented above. I thank R. D. Alexander, L. S. Blumer, D. R. Brooks, G. K. Creighton, P. W. Ewald, W. L. Fink, D. J. Hafner, S. Hatfield, L. R. Heaney, J. L. Hoogland, T. H. Hubbell, J. A. King, A. G. Kluge, G. V. Lauder, B. L. Lundrigan, J. O. Murie, P. Myers, M. Robertson, and R. S. Voss for reading and commenting on drafts of the manuscript. A discussion with G. R. Michener was very helpful. J. D. Kjelgaard drew the figure. Kananaskis Centre, University of Calgary, provided facilities for preparation of the manuscript during my field season.

\section{Literature Cited}

Alexander, R. D., J. L. Hoogland, R. D. Howard, K. M. Noonan, AND P. W. Sherman. 1979. Sexual dimorphisms and breeding systems in pinnipeds, ungulates, primates, and humans, pp. 402-435. In N. A. Chagnon and W. G. Irons (eds.), Evolutionary Biology and Human Social Behavior. Duxbury Press, North Scituate, MA.

BlACK, C. C. 1963. A review of the North American Tertiary Sciuridae. Bull. Mus. Comp. Zool. 130:109-248.

Blumer, L. S. 1979. Male parental care in the bony fishes. Quart. Rev. Biol. 54:149-161.

BRADBURY, J. W. 1981. The evolution of leks, pp. 138-169. In R. D. Alexander and D. W. Tinkle (eds.), Natural Selection and Social Behavior. Chiron, N.Y.

Brooks, D. R. 1985. Historical ecology: A new approach to studying the evolution of ecological associations. Ann. Missouri Bot. Gard. In press.

BRyant, M. D. 1945. Phylogeny of nearctic Sciuridae. Amer. Midl. Natur. 33:257-390.

DoBson, F. S. 1982. Competition for mates and predominant juvenile male dispersal in mammals. Anim. Behav. 30:1183-1192.

- 1984. Environmental influences on sciurid mating systems, pp. 229-249. In J. O. Murie and G. R. Michener (eds.), The Biology of Ground Dwelling Squirrels. Univ. Nebraska Press, Lincoln.

Eldredge, N., And J. Cracraft. 1980. Phylogenetic Patterns and the Evolutionary Process. Columbia Univ. Press, N.Y.

Felsenstein, J. 1985. Phylogenies and the comparative method. Amer. Natur. 125:1-15.

GreEnwood, P. J. 1980. Mating systems, philopatry and dispersal in birds and mammals. Anim. Behav. 28:1140-1162.

Hight, M. E., M. Goodman, And W. Prychodko. 1974. Immunological studies of the Sciuridae. Syst. Zool. 23:12-25.

KING, J. A. 1955. Social behavior, social orga- 
nization, and population dynamics in a blacktailed prairiedog town in the Black Hills of South Dakota. Contrib. Lab. Vert. Biol. 67:1-123.

KreBS, C. J. 1978. A review of the Chitty hypothesis of population regulation. Can. J. Zool. 56:2463-2480.

Krebs, C. J., AND J. H. MYers. 1974. Population cycles in small mammals. Adv. Ecol. Res. 8: 267-399.

LAUDER, G. V. 1981. Form and function: Structural analysis in evolutionary morphology. $\mathrm{Pa}$ leobiol. 7:430-442.

-1982. Historical biology and the problem of design. J. Theoret. Biol. 97:57-67.

LIDICKER, W.Z., JR. 1978. Regulation of numbers in small mammal populations-historical reflections and a synthesis, pp. 122-141. In D. P. Snyder (ed.), Populations of Small Mammals Under Natural Conditions. Pymatuning Laboratory of Ecology, Special Publication No. 5. Univ. Pittsburgh, Pittsburgh, PA.

Lillegraven, J. A. 1974. Biogeographical considerations of the marsupial-placental dichotomy. Ann. Rev. Ecol. Syst. 5:263-283.

- 1975. Biological considerations of the marsupial-placental dichotomy. Evolution 29: 707-722.

Low, B. S. 1978. Environmental uncertainty and the parental strategies of marsupials and placentals. Amer. Natur. 112:197-213.

MitTeR, C., AND D. R. BROOKS. 1983. Phylogenetic aspects of coevolution, pp. 65-98. In D.
J. Futuyma and M. Slatkin (eds.), Coevolution. Sinauer, N.Y.

NADLER, C. F., R. S. HoFFMANN, AND J. J. Pizzimenti. 1971. Chromosomes and serum proteins of prairie dogs and a model of Cynomys evolution. J. Mammal. 52:545-555.

Ralls, K. 1976. Mammals in which females are larger than males. Quart. Rev. Biol. 51:245276.

RIDLEY, M. 1983. The Explanation of Organic Diversity. Oxford Univ. Press, N.Y.

STEARNS, S. C. 1977. The evolution of life history traits. Ann. Rev. Ecol. Syst. 8:145-171.

-1983. The influence of size and phylogeny on patterns of covariation among life-history traits in the mammals. Oikos 41:173-187.

- 1984. The effects of size and phylogeny on patterns of covariation in the life history traits of lizards and snakes. Amer. Natur. 123:56-72.

VItT, L. J., AND R. A. Seigel. 1985. Life history traits of lizards and snakes. Amer. Natur. 125: 480-484.

WILEY, E. O. 1981. Phylogenetics. Wiley, N.Y.

WITTENBERGER, J. F. 1976. The ecological factors selecting for polygyny in altricial birds. Amer. Natur. 110:779-799.

Wittenberger, J. F., AND R. L. Tilson. 1980. The evolution of monogamy: Hypotheses and evidence. Ann. Rev. Ecol. Syst. 11:197-232.

Corresponding Editor: A. E. Dunham

\title{
FOUNDER-FLUSH SPECIATION: AN UPDATE OF EXPERIMENTAL RESULTS WITH DROSOPHILA
}

\author{
Diane M. B. Dodd' AND JefFrey R. Powell \\ Department of Biology, Yale University, P.O. Box 6666, New Haven, CT 06511
}

Received September 28, 1984. Accepted June 7, 1985

Mayr (1954) emphasized the possible causal role of small populations, his founder effects, in initiating speciation. Carson and colleagues rekindled interest in the role of founder events in speciation by their studies of Hawaiian Drosophila (Carson, 1970; Carson et al., 1970; Carson and Yoon, 1982). Basically, Carson hypothesized a causal relationship between two remarkable observations: 1) Despite its relatively young age, the Hawaiian archipelago contains an extraordinarily large number of endemic species of Drosophila; and 2) Chromo-

\footnotetext{
${ }^{1}$ Present address: Department of Biological Sciences, University of North Carolina, Wilmington, NC 28403.
}

somal studies indicate that many species can be traced to ancestral species, and the derived species' chromosomal constitution is best explained by a founder event. Carson has developed a rather specific and comprehensive view of how founder events can promote or cause speciation (see Carson, 1982, and references therein). One aspect of this model is the possible importance of chromosomal rearrangements (e.g., inversions) in controlling rates of recombination. In structurally heterozygous populations, highly coadapted polygenic complexes build up; they become a closed system in the sense that recombination is rare; and, when recombination occurs, the resultant scrambled chromosomes are maladapted. In a founder event chromosomal homozygosity may suddenly be achieved, and the 